\title{
Prognostic factors in prostate and testis cancer
}

\author{
J.P. VAN BRUSSEL and G.H.J. MICKISCH \\ Department of Urology, Erasmus University and Academic Hospital Rotterdam, Rotterdam, the Netherlands
}

\section{Introduction}

The medical management of patients with prostate or testis cancer is based on knowledge of the biological behaviour of these malignancies, as well as factors such as the patient's age and general medical condition. Depending on the expected prognosis, a patient may be treated with curative intent, at times enduring considerable morbidity from the treatment. In other cases, a palliative approach, based on quality-of-life issues, may be the treatment of first choice. It is a challenging task to choose the optimal therapy for an individual patient because of difficulties in predicting precisely the course of the malignant process in each patient. Studies on tissue-related prognostic factors form an extensive part of the contemporary oncological literature. Such studies offer an insight to the molecular pathogenesis of malignancies, potentially improving the power to predict the clinical course and thus assisting in clinical decisionmaking. In this review, we discuss the clinical use of tissue-related prognostic factors in prostate and testis cancer and assess the published literature on experimental prognostic markers.

\section{Prostate cancer}

Prostate cancer is the commonest cancer in western men and is second only to lung cancer as a cause of cancer death [1,2]. As the overall lifetime probability of developing clinically evident prostate cancer is much lower than the prevalence at autopsy by the age of 80 years $(16 \%$ vs $80 \%)$, most men die with rather than from prostate cancer. However, it is clinically important to distinguish the potentially lethal from the relatively indolent tumours. The use of prognostic markers may be crucial to identify the subgroup of patients with aggressive prostate cancer. We will discuss both clinically applied markers and experimental markers.

\section{Stage}

The TNM system is the one most commonly used for clinical and pathological staging of prostate cancer [3]; it involves determining the anatomical extent of the tumour. Currently, it is associated with problems of understaging and sometimes overstaging, as shown by pathological examination of resected specimens [4]. This creates uncertainty and controversy about the comparative efficacy of different treatments and expected outcomes for patients. Standardization of staging procedures after radical prostatectomy (RP) and clearly defining the extraprostatic extension and positive surgical margins will result in significant improvements in staging. Additional data to enhance the accuracy of staging and prediction of clinical outcome for individual patients may include; systematic biopsies to predict preoperative tumour volume and a volume-based prognostic index, linking cancer volume with extraprostatic extension, seminal vesicle invasion and lymph node metastases [5]. A multiple prognostic index [6], including variables such as serum PSA, tumour volume, tumour grade, tumour localization and DNA content, may have more predictive power than conventional staging methods alone.

\section{Grade}

The Gleason grading system is currently the most widely used system for grading prostate cancer and is a powerful variable in the management of patients with this condition. It takes into account the histological tumour heterogeneity of prostate cancer. This feature and tumour multifocality can result in significant sampling errors when grading is established from needle-core biopsies [7,8]. Furthermore, the prognostic power of grade is greatest in tumours with the lowest (2-4) or highest (8-10) scores. However, most tumours have an intermediate score (5-7) and show strikingly heterogeneous biological aggressiveness. The Gleason score cannot discriminate the indolent from the more aggressive cancers. Also, inter- and intra-observer variability handicap the use of the Gleason score as a prognosticator in patients with prostate cancer.

\section{PSA}

Since its discovery in 1971 and its purification from seminal plasma and the prostate in 1979, PSA has been widely used as a tumour marker for screening, diagnosis and monitoring of prostate cancer [9]. Although the name suggests otherwise, PSA is not produced exclusively by the prostate but has been detected in urine, the periurethral glands, peri-anal glands, saliva, amniotic 
fluid, milk of lactating women and serum of some women with renal cancer. PSA is not strictly organ-specific, nor is it disease-specific; elevated PSA levels can be found in a small proportion of normal men and in many men with $\mathrm{BPH}$ and prostatitis. Although PSA is not a perfect marker, many publications have confirmed the value of serum PSA level as a prostate cancer marker and as a surrogate endpoint for studies of locally confined prostate cancer [10]. The role of PSA as a predictor of outcome in prostate cancer has been proposed by several investigators [11]. Tissue PSA has been the subject of fewer reports, although its prognostic value has been studied in biopsy specimens [12-14] and TUR specimens [15] with T1b prostate cancer, indicating that PSA immunohistochemistry could predict clinical outcome. Most studies have reported that tissue and serum PSA levels are correlated. As serum PSA is considerably easier to obtain, its value as a prognostic marker is greater than tissue PSA. However, undifferentiated androgen-unresponsive prostate cancer cells are unable to produce PSA, possibly explaining the lack of PSA increase in a proportion of patients with hormone-refractory disease. Novel applications of PSA, such as PSA density, PSA slope, PSA doubling time and free PSA have been derived and have, to a certain extent, improved the sensitivity and specificity of PSA testing [16].

Recently, research has focused on detecting circulating PSA-producing cells using RT-PCR to detect PSA-mRNA. In one study, the presence of circulating PSA-mRNA was of independent prognostic value in predicting PSA progression after RP [17]. Positive results should be interpreted cautiously as the PSA gene is not uniquely expressed in prostate cells and the clinical significance of single circulating tumour cells is unknown.

\section{Prostate-specific membrane antigen (PSMA)}

PSMA is a relatively new marker for prostate cancer [18] and is expressed by most intra-epithelial neoplasms as well as primary and metastatic prostate cancer specimens. PSMA is also expressed at detectable levels in small intestine, brain and salivary gland [19]. At present, the role of PSMA as a prognostic marker for prostate cancer is inconclusive. The expression of PSMA was high in poorly differentiated and metastatic cancer specimens, but did not correlate with nodal status, seminal vesicle invasion or extraprostatic extension. The ratio of PSMA and PSMA', an alternatively spliced variant of PSMA mRNA, may be a useful index for assessing prostate cancer progression [20]. Studies of serum PSMA reported that serum PSMA levels may serve as a prognostic marker for prostate cancer [21-23]. PSMA RT-PCR assays have been developed to detect circulating PSMAproducing cells in the blood; the sensitivity appears to be inferior to the PSA RT-PCR assay [24] but this assay requires further study.

\section{Epithelial cadherins}

Epithelial cadherin (E-cadherin) is a calcium-dependent cell adhesion molecule that plays an important role in maintaining the epithelial phenotype. Decreased expression of E-cadherin has been detected in several carcinomas, and implies disordered intercellular attachment and increased risk of invasion and metastasis [25]. The role of E-cadherin in prostate cancer was reported in studies of the Dunning rat prostate cancer, in which E-cadherin was expressed by normal rat prostate tissue and noninvasive tumours but was undetectable in invasive rat prostate cancer sub-lines [26]. In human prostate cancer an inverse correlation between E-cadherin expression and grade, stage and overall survival has been reported, suggesting a role for E-cadherin as a prognosticator in prostate cancer [25]. More studies are needed to confirm these findings and to determine the precise clinical importance of E-cadherin.

\section{DNA content (ploidy)}

Tumour DNA content has been the subject of numerous studies of prostate cancer (reviewed by Adolfsson [27]). Many of these studies have suggested that the DNA aneuploidy of RP specimens and needle biopsies correlates with progression, metastasis and poor prognosis, although conflicting data have been presented. The clinical applicability of tumour ploidy measurements has been discussed in reports by the WHO [28] and the College of American Pathologists [29]. The former group advised the use of ploidy assessments for $\mathrm{T} 2$ and T3 tumours, whereas the latter group concluded that DNA ploidy may be useful in patients with stage T3 or $\mathrm{pN}+$ tumours who are subsequently treated with adjuvant hormonal therapy. DNA ploidy has potential as a prognostic marker, but its clinical role remains a matter of debate. Phase-III clinical trials are needed to clarify this issue and to prove that ploidy status provides information beyond stage and grade. In addition, technical issues, involving DNA determination by image analysis, need to be resolved to allow such studies to proceed.

\section{Nuclear morphometry}

Measurement of nuclear roundness, size, form and texture of nuclei may have prognostic value in patients with prostate cancer [30]. However, difficulties with image analysis techniques, tissue handling and reproducibility of results remain a major concern and have 
prevented further evaluation of nuclear morphometry as a prognosticator in prostate cancer patients.

\section{2-lipoxygenase}

12-lipoxygenase (12-LOX) is responsible for the production of 12(S)-hydroxy-eicosatetraenoic acid [12(S)HETE] which plays a role in invasion and metastasis of cancer [31]. 12-LOX may serve as a correlative marker for prostate cancer with an aggressive phenotype and poor prognosis, as shown by RT-PCR and in situ hybrid ization in a study comparing expression of 12-LOX in prostate cancer tissues of different stages [31].

\section{P53 tumour-suppressor gene}

Wild-type p53 protein is a suppressor of cell growth and transformation, causing inhibition of cell proliferation by G1 arrest and, under certain circumstances, apoptosis. Mutation on the $p 53$ gene, which is located on the short arm of chromosome 17 , is the most common alteration in cancers. Loss of wild-type p53 function can lead to deregulation of the cell cycle and DNA replication, defective DNA repair, and selective growth advantage, resulting in tumour formation and progression [32]. The role of p53 and its prognostic value in human prostate cancer remains unclear. A correlation between mutation of the p53 gene and tumour progression has been reported in several studies, with mutations occurring as a late event in the development of prostate cancer $[33,34]$. However, other studies did not confirm this correlation [35] and suggested that p53 abnormalities may be an early event in prostate cancer progression [36]. Larger well-defined groups of patients must be studied to assess definitively the prognostic value of p53 in prostate cancer. Standardization of a method of assessing p53 also remains an issue.

\section{Tumour proliferative activity}

Prostate cancers generally have low levels of proliferation. Different techniques can be used to assess cellular proliferation, including flow cytometry, determination of the S-phase fraction, bromodeoxyuridine labelling and immunostaining for proliferating cell nuclear antigen (PCNA) and Ki-67. The potential prognostic value of proliferation has been examined by several groups, with conflicting results. Some have reported a positive correlation with grade and stage [37], while others have not [38]. Heterogeneity within a tumour poses problems in interpreting the results. At present, inconsistent results hamper the clinical application of proliferative activity as a prognosticator.

\section{Apoptosis}

Apoptosis, or programmed cell death, may be an important factor in the response to therapy of prostate cancer and for prognosis. The bcl-2 protein is able to block the apoptotic pathway [39]. In normal prostate tissue bcl-2 is expressed in the basal cell layer of glands, but not in the secretory cells. In hormone-refractory prostate cancer the expression of bcl-2 is markedly increased [40] suggesting that elevated levels of this protein may provide prostate cancer cells with the ability to survive in an environment with no androgens. Transfection of bcl-2 into the hormone-dependent prostate cancer cell line LNCaP enabled these cells to survive in an androgen-depleted milieu [41]. In one study, bcl-2 had prognostic value using univariate analysis when examining RP samples [42] and in a further study was an independent prognosticator for time to PSA progression [43]. As apoptosis involves many other proteins (such as p53, bax and bcl-X) and is related to proliferation, prognostic marker studies should be conducted combining these factors.

\section{Neuroendocrine differentiation}

Neuroendocrine cells are components of prostatic ducts and acini, as well as prostate cancer with neuroendocrine differentiation, which may show resistance to hormonal therapy [44]. However, most studies report no significant prognostic relevance [45].

\section{Other prognostic markers}

The number of studies published on prognostic markers for prostate cancer is rapidly increasing. Many new experimental markers have been described, and their clinical importance and applicability as prognosticators remain to be determined. These markers include microvessel density (angioneogenesis) [46], TGF $\beta 1$ [47] oligosaccharide sialyl Lewis-X [48], CD44 [49] and oncoantigen 519 [50].

\section{Conclusion}

A vast number of publications have appeared on prognostic markers for prostate cancer, discussing both clinically useful and experimental markers [51,52]. Clinical and pathological stage (TNM classification), histological grade (Gleason score) and serum PSA, with its molecular variants, are the most powerful prognostic factors in prostate cancer. However, at present, there are no tumour markers that can reliably predict the clinical course of prostate cancer for an individual patient. Further efforts are needed in the search for the ideal prognostic marker for prostate cancer. 


\section{Testicular cancer}

Testicular cancer has a relatively good prognosis, even in an advanced stages. Cure rates for disseminated germ cell tumours of $\geqslant 90 \%$ have been reported in patients undergoing cisplatin-based chemotherapy [53]. Improvements in staging methods, surgical techniques and radiotherapy have also contributed to this favourable prognosis.

Nevertheless, the optimal management of patients with locally confined disease remains to be defined. Morbidity and toxicity from treatment regimens must be reduced, while maintaining high cure rates. Furthermore, it is important to identify those patients who are at risk of metastatic relapse. If the risk of metastasis could be determined using prognostic factors, an individually designed therapy could be offered to the patient.

In patients with advanced nonseminomatous testicular cancer complete remission rates are still low in some subgroups. Despite considerable progress since the introduction of cisplatin-based chemotherapy, $\sim 20 \%$ of patients presenting with metastases die from their disease [54]. In this group, the goal of investigational trials is to improve the proportion of complete remissions while accepting concurrent toxicity. The application of prognostic factors might help to define a more individual treatment schedule for these patients, resulting in increased cure rates and diminished side-effects of therapy.

Among urological malignancies, testicular cancer takes a special place because clinical decision-making is largely based on the application of prognostic factors. To assess the risk for metastatic testicular cancer the most important prognostic features are the extent of the disease and serum determination of tumour markers.

\section{Histological prognostic factors}

Histological prognostic factors include the percentage of embryonal carcinoma within the primary tumour [55], and vascular invasion, i.e. the infiltration of testicular veins by the tumour. In a recent study by Lewis et al. [56], the presence of embryonal carcinoma within the primary tumour, in addition to vascular invasion, appeared to be an independent prognostic factor for tumour progression, and the prognostic relevance was prospectively confirmed [57].

\section{Serum tumour markers}

AFP, a foetal serum-binding protein, is normally found in minimal amounts $(<100 \mathrm{ng} / \mathrm{L})$ after the first year of life [58]. It is elevated in $50-70 \%$ of patients with testicular cancers, including yolk sac and embryonal tumours, or combinations of these tumours [59]. Patients with pure seminoma do not have elevated AFP levels. On complete tumour excision, serum AFP levels may return to normal in $\approx 1$ month, depending on the peak serum concentration and AFP half-life.

Another marker, hCG, is normally produced by syncytiotrophoblasts of the placenta. Normal males have serum levels of the beta subunit $(\beta-\mathrm{hCG})$ of $<5 \mathrm{mIU} / \mathrm{mL}$, but $\beta$-hCG levels are elevated in $40-60 \%$ of patients with testicular cancer. $\beta$-hCG levels are high in all cases of choriocarcinoma, $80 \%$ of cases of embryonal cell carcinoma and $10-25 \%$ of cases of pure seminoma [59]. Levels return to normal values normally about a week after radical tumour excision, depending on the peak serum concentration and the half-life of $\beta$-hCG. Elevated levels of AFP and $\beta$-hCG are not specific for testicular cancer and may occur with benign liver disease, and with lung, gastric, pancreatic, hepatocellular cancer (AFP) and breast, bladder, pancreatic, gastric, hepatocellular and lung cancer ( $\beta$-hCG) [58].

The enzyme $\mathrm{LDH}$ is responsible for lactic acid oxidation in muscle, liver and kidneys. The level of isoenzyme 1 is elevated in most patients with advanced testicular cancers and particularly in those with large-volume tumours [58].

Recently, international consensus on the stratification of patients with testicular cancer has been reached by the International Germ Cell Cancer Collaborative Group (IGCCCG), based on data from multiple clinical trials in Europe, North America and Australia (reviewed by McCaffrey [54]). This group found that pretreatment levels of AFP, hCG and LDH, the site of the primary tumour and the presence of nonpulmonary visceral metastases were independent prognosticators for survival. Using this classification, patients with nonseminomatous germ cell tumours (NSGCTs) can be stratified into three groups based on response to chemotherapy (Table 1). Patients with seminoma patients could be divided in two risk groups; one with 'good risk', with no evidence of nonpulmonary metastases, and an intermediate risk group with evidence of nonpulmonary metastases. This consensus has resulted in a new staging system for testis cancer, that incorporates both serum markers and anatomical features, which is clinically relevant to the selection of patients for optimal treatment.

\section{Experimental prognostic factors}

The prognostic value of DNA index, S-phase fraction and proliferation index in NSGCTs has been reported [60], but results are conflicting; in multivariate analysis these factors were not superior to histological prognostic factors such as vascular invasion and the presence of embryonal carcinoma cells. 


\begin{tabular}{|c|c|c|c|c|}
\hline $\begin{array}{l}\text { Risk } \\
\text { group }\end{array}$ & $\begin{array}{l}\text { LDH } \\
\times \text { normal }\end{array}$ & $\begin{array}{l}h C G \\
(I U / m L)\end{array}$ & $\begin{array}{l}A F P \\
(\mu g / m L)\end{array}$ & $\begin{array}{l}\text { Evidence of } \\
\text { nonpulmonary } \\
\text { metastases }\end{array}$ \\
\hline Good & $<1.5$ & $<5$ & $<1$ & No \\
\hline Intermediate & $1.5-10$ & $5-50$ & $1-10$ & No \\
\hline Poor & $>10$ & $>50$ & $>10$ & Yes \\
\hline
\end{tabular}

Table 1 International Germ Cell Cancer Collaborative Group; nonseminomatous germ cell tumour risk groups
Molecular analysis of testicular tumours has lead to the detection of genetic alterations that are associated with malignant transformation. Isochromosome $12 \mathrm{p}$ (i[12p]) has been identified in $80 \%$ of nonseminomas (and only $7 \%$ of seminomas) and may represent a marker for early tumorigenesis [61]; it has been associated with tumour stage and prognosis [62]. There is an increased frequency of chromosomal alteration at chromosome 10p13, 7q11.2 and 12p11q13 in metastases [63]. Amplification of the hst-1 proto-oncogene has been reported to correlate with advanced tumour stage [64]. However, much work is needed in the molecular biology of testicular cancer to provide proof of the value of these variables as prognosticators for testicular cancer.

\section{Conclusion}

Although the use of a new staging system for testis cancer is a major advance in predicting the outcome of treatment, a few 'good-risk' patients will die of multidrugresistant disease, despite favourable prognostic features, and about a third of 'poor-risk' patients survive. Therefore, there remains a need to identify further independent prognostic factors which will help increase the prognostic power of the current staging system.

\section{References}

1 Parker SL, Tong T, Bolden S, Wingo PA. Cancer statistics, 1996. CA Cancer J Clin 1996; 46: 5-27

2 van der Gulden JW, Kiemeney LA, Verbeek AL, Straatman H. Mortality trend from prostate cancer in The Netherlands (1950-89). Prostate 1994; 24: 33-8

3 Schroder FH, Hermanek P, Denis L, Fair WR, Gospodarowicz MK, Pavone-Macaluso M. The TNM classification of prostate cancer. Prostate 1992; 4 (Suppl): 129-38

4 Bostwick DG. Staging prostate cancer-1997: current methods and limitations. Eur Urol 1997; 32: 2-14

5 Bostwick DG, Graham SD Jr, Napalkov P et al. Staging of early prostate cancer: a proposed tumor volume-based prognostic index. Urology 1993; 41: 403-11

6 Fielding LP, Fenoglio-Preiser CM, Freedman LS. The future of prognostic factors in outcome prediction for patients with cancer. Cancer 1992; 70: 2367-77

7 Gleason DF. Histologic grading of prostate cancer: a perspective. Hum Pathol 1992; 23: 273-9
8 Bostwick DG. Gleason grading of prostatic needle biopsies. Correlation with grade in 316 matched prostatectomies. Am J Surg Pathol 1994; 18: 796-803

9 Oesterling JE. Prostate specific antigen: a critical assessment of the most useful tumor marker for adenocarcinoma of the prostate. J Urol 1991; 145: 907-23

10 Partin AW, Pound CR, Clemens JQ, Epstein JI, Walsh PC. Serum PSA after anatomic radical prostatectomy. The Johns Hopkins experience after 10 years. Urol Clin North Am 1993; 20: 713-25

11 Blackledge GR, Lowery K. Role of prostate-specific antigen as a predictor of outcome in prostate cancer. Prostate 1994; 5 (Suppl): 34-8

12 Stege R, Tribukait B, Lundh B, Carlstrom K, Pousette A, Hasenson M. Quantitative estimation of tissue prostate specific antigen, deoxyribonucleic acid ploidy and cytological grade in fine needle aspiration biopsies for prognosis of hormonally treated prostatic carcinoma. J Urol 1992; 148: 833-7

13 Sakai H, Yogi Y, Minami Y, Yushita Y, Kanetake H, Saito Y. Prostate specific antigen and prostatic acid phosphatase immunoreactivity as prognostic indicators of advanced prostatic carcinoma. J Urol 1993; 149: 1020-3

14 Berner A, Harvei S, Tretli S, Fossa SD, Nesland JM. Prostatic carcinoma: a multivariate analysis of prognostic factors. Br J Cancer 1994; 69: 924-30

15 Mohler JL, Partin AW, Epstein JI et al. Prediction of prognosis in untreated stage A2 prostatic carcinoma. Cancer 1992; 69: 511-9

16 Lee CT, Oesterling JE. Diagnostic markers of prostate cancer: utility of prostate-specific antigen in diagnosis and staging. Semin Surg Oncol 1995; 11: 23-35

17 Olsson CA, de Vries GM, Raffo AJ et al. Preoperative reverse transcriptase polymerase chain reaction for prostate specific antigen predicts treatment failure following radical prostatectomy. J Urol 1996; 155: 1557-62

18 Horoszewicz JS, Kawinski E, Murphy GP. Monoclonal antibodies to a new antigenic marker in epithelial prostatic cells and serum of prostatic cancer patients. Anticancer Res 1987; 7: 927-35

19 Troyer JK, Beckett ML, Wright GL Jr Detection and characterization of the prostate-specific membrane antigen (PSMA) in tissue extracts and body fluids. Int $J$ Cancer 1995; 62: 552-8

20 Su SL, Huang IP, Fair WR, Powell CT, Heston WD. Alternatively spliced variants of prostate-specific membrane antigen RNA. ratio of expression as a potential measurement of progression. Cancer Res 1995; 55: 1441-3 
21 Murphy G, Ragde H, Kenny G et al. Comparison of prostate specific membrane antigen, and prostate specific antigen levels in prostatic cancer patients. Anticancer Res 1995; 15: $1473-9$

22 Murphy GP, Holmes EH, Boynton AL et al. Comparison of prostate specific antigen, prostate specific membrane antigen, and LNCaP-based enzyme-linked immunosorbent assays in prostatic cancer patients and patients with benign prostatic enlargement. Prostate 1995; 26: 164-8

23 Israeli RS, Miller WH Jr, Su SL et al. Sensitive nested reverse transcription polymerase chain reaction detection of circulating prostatic tumor cells: comparison of prostatespecific membrane antigen and prostate-specific antigenbased assays. Cancer Res 1994; 54: 6306-10

24 Cama C, Olsson CA, Raffo AJ et al. Molecular staging of prostate cancer. II. A comparison of the application of an enhanced reverse transcriptase polymerase chain reaction assay for prostate specific antigen versus prostate specific membrane antigen. J Urol 1995; 153: 1373-8

25 Umbas R, Schalken JA, Aalders TW et al. Expression of the cellular adhesion molecule E-cadherin is reduced or absent in high-grade prostate cancer. Cancer Res 1992; 52: 5104-9

26 Bussemakers MJ, van Moorselaar RJ, Giroldi LA et al. Decreased expression of E-cadherin in the progression of rat prostatic cancer. Cancer Res 1992; 52: 2916-22

27 Adolfsson J. Prognostic value of deoxyribonucleic acid content in prostate cancer: a review of current results. Int J Cancer 1994; 58: 211-6

28 Schröder F, Tribukait B, Böcking A et al. Clinical utility of cellular DNA measurements in prostatic carcinoma. Scand J Urol Nephrol 1994; 162: 51-64

29 Grignon DJ, Hammond EH. College of American Pathologists Conference XXVI on clinical relevance of prognostic markers in solid tumors. Report of the Prostate Cancer Working Group. Arch Pathol Lab Med 1995; 119: $1122-6$

30 Blom JH, Ten Kate FJ, Schroeder FH, van der Heul RO. Morphometrically estimated variation in nuclear size. A useful tool in grading prostatic cancer. Urol Res 1990; 18: $93-9$

31 Gao X, Honn K. 12-Lipoxygenase and 12(S)-HETE in cancer invasion and metastasis. Adv Oncol 1995; 11: 3-8

32 Nigro JM, Baker SJ, Preisinger AC et al. Mutations in the p53 gene occur in diverse human tumour types. Nature 1989; 342: 705-8

33 Effert PJ, McCoy RH, Walther PJ, Liu ET. p53 gene alterations in human prostate carcinoma. J Urol 1993; 150: 257-61

34 Bookstein R, MacGrogan D, Hilsenbeck SG, Sharkey F, Allred DC. p53 is mutated in a subset of advanced-stage prostate cancers. Cancer Res 1993; 53: 3369-73

35 Dinjens WN, van der Weiden MM, Schroeder FH, Bosman FT, Trapman J. Frequency and characterization of p53 mutations in primary and metastatic human prostate cancer. Int J Cancer 1994; 56: 630-3

36 Meyers FJ, Chi SG, Fishman JR, deVere White RW, Gumerlock PH. p53 mutations in benign prostatic hyperplasia. J Natl Cancer Inst 1993; 85: 1856-8
37 Spires SE, Banks ER, Davey DD, Jennings CD, Wood DP Jr, Cibull ML. Proliferating cell nuclear antigen in prostatic adenocarcinoma: correlation with established prognostic indicators. Urology 1994; 43: 660-6

38 Bubendorf L, Sauter G, Moch H et al. Ki67 labelling index: an independent predictor of progression in prostate cancer treated by radical prostatectomy. J Pathol 1996; 178: 437-41

39 Hockenbery D, Nunez G, Milliman C, Schreiber RD, Korsmeyer SJ. Bcl-2 is an inner mitochondrial membrane protein that blocks programmed cell death. Nature 1990; 348: 334-6

40 McDonnell TJ, Troncoso P, Brisbay SM et al. Expression of the protooncogene bcl-2 in the prostate and its association with emergence of androgen-independent prostate cancer. Cancer Res 1992; 52: 6940-4

41 Raffo AJ, Perlman H, Chen MW, Day ML, Streitman JS, Buttyan R. Overexpression of bcl-2 protects prostate cancer cells from apoptosis in vitro and confers resistance to androgen depletion in vivo. Cancer Res 1995; 55: 4438-45

42 Bubendorf L, Sauter G, Moch H et al. Prognostic significance of Bcl-2 in clinically localized prostate cancer. Am J Pathol 1996; 148: 1557-65

43 Bauer JJ, Sesterhenn IA, Mostofi FK, McLeod DG, Srivastava S, Moul JW. Elevated levels of apoptosis regulator proteins $\mathrm{p} 53$ and bcl-2 are independent prognostic biomarkers in surgically treated clinically localized prostate cancer. J Urol 1996; 156: 1511-6

44 di Sant'Agnese PA. Neuroendocrine differentiation in human prostatic carcinoma. Hum Pathol 1992; 23: 287-96

45 Noordzij MA, van der Kwast TH, van Steenbrugge GJ, Hop WJ, Schroder FH. The prognostic influence of neuroendocrine cells in prostate cancer: results of a long-term followup study with patients treated by radical prostatectomy. Int J Cancer 1995; 62: 252-8

46 Weidner N, Carroll PR, Flax J, Blumenfeld W, Folkman J. Tumor angiogenesis correlates with metastasis in invasive prostate carcinoma. Am J Pathol 1993; 143: 401-9

47 Eastham JA, Truong LD, Rogers E et al. Transforming growth factor-beta 1: comparative immunohistochemical localization in human primary and metastatic prostate cancer. Lab Invest 1995; 73: 628-35

48 Jorgensen T, Berner A, Kaalhus O, Tveter KJ, Danielsen HE, Bryne M. Up-regulation of the oligosaccharide sialyl LewisX: a new prognostic parameter in metastatic prostate cancer. Cancer Res 1995; 55: 1817-9

49 Noordzij MA, van Steenbrugge GJ, Verkaik NS, Schröder $\mathrm{FH}$, van der Kwast TH. The prognostic value of CD44 isoforms in prostate cancer patients treated by radical prostatectomy. Clin Cancer Res 1997; 3: 805-15

50 Epstein JI, Carmichael M, Partin AW. OA-519 (fatty acid synthase) as an independent predictor of pathologic state in adenocarcinoma of the prostate. Urology 1995; 45: 81-6

51 Gao X, Porter AT, Grignon DJ, Pontes JE, Honn KV. Diagnostic and prognostic markers for human prostate cancer. Prostate 1997; 31: 264-81

52 Noordzij MA, van der Kwast TH, van Steenbrugge GJ, Schröder FH. The application of prognostic tissue markers 
to prostate cancer. Clinical and experimental studies. Doctoral Thesis, Rotterdam: Erasmus University 1997: $11-43$

53 Stoter G, Vendrik CP, Struyvenberg A et al. Five-year survival of patients with disseminated nonseminomatous testicular cancer treated with cisplatin, vinblastine, and bleomycin. Cancer 1984; 54: 1521-4

54 McCaffrey JA, Bajorin DF, Motzer RJ. Risk assessment for metastatic testis cancer. Urol Clin North Am 1998; 25: 389-95

55 Wishnow KI, Johnson DE, Swanson DA et al. Identifying patients with low-risk clinical stage I nonseminomatous testicular tumors who should be treated by surveillance. Urology 1989; 34: 339-43

56 Lewis DJ, Sesterhenn IA, McCarthy WF, Moul JW. Immunohistochemical expression of P53 tumor suppressor gene protein in adult germ cell testis tumors: clinical correlation in stage I disease. J Urol 1994; 152: 418-23

57 Read G, Stenning SP, Cullen MH et al. Medical Research Council prospective study of surveillance for stage I testicular teratoma. Medical Research Council Testicular Tumours Working Party. J Clin Oncol 1992; 10: 1762-8

58 Klein EA. Tumor markers in testis cancer. Urol Clin North Am 1993; 20: 67-73

59 Vugrin D, Friedman A, Whitmore WF Jr Correlation of serum tumor markers in advanced germ cell tumors with responses to chemotherapy and surgery. Cancer 1984; 53: $1440-5$

60 Moul JW, Foley JP, Hitchcock CL et al. Flow cytometric and quantitative histological parameters to predict occult disease in clinical stage I nonseminomatous testicular germ cell tumors. J Urol 1993; 150: 879-83

61 Atkin NB, Baker MC. Specific chromosome change, i(12p), in testicular tumours? Lancet 1982; 2: 1349

62 Bosl GJ, Dmitrovsky E, Reuter VE et al. Isochromosome of the short arm of chromosome 12: clinically useful markers for male germ cell tumors. J Natl Cancer Inst 1989; 81: $1874-8$

63 Rodriguez E, Mathew S, Reuter V, Ilson DH, Bosl GJ, Chaganti RS. Cytogenetic analysis of 124 prospectively ascertained male germ cell tumors. Cancer Res 1992; 52: 2285-91

64 Strohmeyer T, Peter S, Hartmann M et al. Expression of the hst-1 and c-kit protooncogenes in human testicular germ cell tumors. Cancer Res 1991; 51: 1811-6

\section{Authors}

J.P. van Brussel, M.D., President in Urology. G.H.J. Mickisch, M.D., PhD, Professor of Urology.

Correspondence: Academic Hospital Rotterdam, H1095, Dr Molenwaterplein 40, NL 3015 GD Rotterdam, The Netherlands. E-mail: Mickisch@urol.azr.nL

\section{MCQs}

1. Which statement is correct?

(a) tissue markers have found widespread acceptance in clinical decision making (b) high specificity, high sensitivity and high reproducibility are important issues in the development of clinically useful tissue markers

(c) with increased awareness of cost-effectiveness in clinical decision-making, interest in tissue markers has decreased in recent years

(d) tissue markers that have found widespread clinical acceptance are strictly tumour-specific

2. Which statement is correct?

(a) prostate cancer-specific markers are well established in a clinical routine application

(b) the clinical distinction between a T2 and a T3 stage is a highly reproducible prognosticator in prostate cancer (c) the Gleason grading system for prostate cancer has its highest reliability in intermediate scores (Gleason 5 to 7)

(d) inter- and intra-observer variability handicaps the use of the Gleason score as a prognosticator for prostate cancer

3. Which statement is correct?

(a) PSA values are reliable indicators of progression in hormone-refractory disease

(b) PSA is produced only by benign or cancerous prostate cells

(c) the appearance of PSA-producing cells in the bloodstream is very helpful in clinical decision-making

(d) E-cadherin expression is inversely correlated with grade, stage and overall survival in prostate cancer

4. Which statement is correct?

(a) DNA ploidy is a good prognosticator in stage T1

(b) the value of p53 in prostate cancer as a prognostic factor is still unclear

(c) proliferative activity is established as a valuable prognosticator in prostate cancer

(d) neuroendocrine differentiation is inversely correlated to prognosis in prostate cancer

5. Which statement is correct?

(a) prognostic markers can distinguish between aggressive and indolent tumours

(b) more men die with prostate cancer rather than from prostate cancer

(c) a DRE is more accurate in diagnosing prostate cancer in stage T1c than is the PSA level

(d) PSA values of $<10 \mathrm{ng} / \mathrm{mL}$ excludes the appearance of bone metastasis

6. Which statement is correct?

(a) prostate specific membrane antigen (PSMA) is only expressed by prostatic intraepithelial neoplasia, primary or metastatic prostate cancer

(b) hormone-independent growth of prostate cancer can be achieved by the expression of bcl-2 
(c) expression of CD44 splice variants may be inversely correlated with the prognosis of patients with prostate cancer

(d) determination of proliferative activity has clinical value as a prognosticator for prostate cancer

7. Which statement is correct?

(a) the main goal of current treatment strategies in stage I and II testicular cancer is to attain higher cure rates

(b) the side-effects of therapy in low-stage testicular cancer are minor, if any

(c) primary testicular cancer is refractory to many forms of chemotherapy

(d) patients with seminoma with lung metastasis may belong to a good-risk group

8. Which statement is correct?

(a) elevated levels of AFP and hCG are specific for testicular cancer

(b) large-volume testicular cancer most commonly induces higher levels of LDH isoenzyme 1

(c) amplification of the HST-1 proto-oncogene has been reported to correlate with low-stage testicular cancer

(d) elevation of hCG worsens the prognosis of seminoma
9. Which statement is correct?

(a) an important area for prognostic factors in testicular cancer is to identify patients with a high risk for metastatic relapse

(b) to date, prognostic factors in testicular cancer had little impact on clinical decision-making compared with other urological malignancies

(c) AFP levels may provide a valuable indication of the management of patients with seminoma

(d) hCG levels are increased in half of patients with choriocarcinoma, with a subsequent impact on their prognosis

10. Which statement is correct?

(a) pre-treatment levels of AFP and hCG are not independent prognosticators for survival in patients with NSGCTs

(b) the percentage of embryonal carcinoma within the primary testicular cancer is inversely correlated with the prognosis

(c) an increased frequency of chromosomal alterations is established as a prognosticator in NSGCTs

(d) levels of AFP and $\beta$ hCG after treatment are most useful in clinical-decision making 